Sección monográfica / Monographic section

\title{
"La vida se ha vuelto más feliz, camaradas": la construcción de la cultura del consumo soviética
}

\author{
Life Has Become More Joyous, Comrades: the construction of the \\ Soviet consumer culture ${ }^{1}$
}

\author{
Jukka Gronow (iD) \\ Universidad de Helsinki, Finlandia \\ jukka.gronow@helsinki.fi
}

\section{Sergey Zhuravlev}

Instituto de Historia Rusa de la Academia Rusa de las Ciencias, Moscú, Rusia

svzhuravlev@mail.ru

Recibido / Received: 20/04/2020

Aceptado / Accepted: 26/05/2020

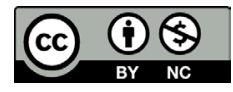

\section{RESUMEN}

En este artículo, el objetivo es el de describir el origen y construcción de la cultura del consumo en la Unión Soviética, prestando atención particular a uno de sus ejemplos icónicos, como es la cultura alimentaria. Nuestro texto se dividirá en tres secciones. En la primera, haremos referencia a la peculiar y original construcción de la sociedad de consumo soviética, en pleno estalinismo. En la segunda, nos concentraremos en el espacio concreto del consumo alimentario, con referencia en particular a dos cuestiones: el desarrollo de los servicios de restauración y algunos de los bienes asociados a esta nueva cultura de consumo, como el champán. Finalmente, la última sección de conclusiones se hará una valoración crítica de esta peculiar forma de sociedad de consumo.

Palabras clave: Cultura de consumo; URSS; socialismo; consumo alimentario; lujo.

\section{ABSTRACT}

In this article, our aim is to describe the emergence and consolidation of the consumer culture in the Soviet Union, paying particular attention to one of its iconic examples: the food culture. Our text will be divided into three sections. Firstly, we will refer to the peculiar and original development of the Soviet consumer society at the height of Stalinism. Secondly, we will focus on the specific space of food consumption, with a reference to two specific issues: the development of catering services and the launching of goods associated with this new culture of consumption, such as champagne. Finally, the concluding remarks will focus on a critical assessment of this peculiar form of consumer society.

Keywords: Consumer culture; USSR; socialism; food consumption; luxury.

\footnotetext{
*Autor para correspondencia / Corresponding author: Jukka Gronow. jukka.gronow@helsinki.fi

Sugerencia de cita / Suggested citation: Gronow, J. y Zhuravlev, S. (2020). "La vida se ha vuelto más feliz, camaradas": la construcción de la cultura del consumo soviética. Revista Española de Sociología, 29 (3, supl. 1), 231-244. https://doi.org/10.22325/fes/res.2020.67

1 Este texto ha sido editado y traducido por Carlos Jesús Fernández Rodríguez a partir de una serie de materiales facilitados por Jukka Gronow, el autor principal de este artículo. Mi agradecimiento a Riie Heikkilä por sus sugerencias en relación a la versión final del texto en castellano (N. del T.).
} 


\section{INTRODUCCIÓN}

Uno de los aspectos más peculiares de la hoy extinta Unión Soviética a ojos de un observador extranjero eran los contrastes existentes en el terreno del consumo. Los pocos turistas y visitantes extranjeros que, a finales de la década de los sesenta, paseaban por Moscú (y de esto puede dar fe uno de los autores de este artículoº ${ }^{2}$ podían visualizar de forma relativamente habitual dos fenómenos contrapuestos. El primero tenía que ver con algo que prácticamente todo ciudadano procedente del Occidente capitalista había escuchado alguna vez, en los medios de comunicación y en las crónicas de las personas que habían conseguido acceder al opaco universo de la URSS: la escasez y mala calidad de los bienes de consumo. Las largas colas que se formaban ante las generalmente desabastecidas tiendas moscovitas eran, sin duda, una de las estampas más conocidas del mundo soviético, y estas de alguna manera representaban, en el mundo capitalista, el ejemplo visible de las disfunciones de las economías socialistas. La calidad de los bienes que se encontraban a la venta era, además, muy inferior a los estándares habituales occidentales, con escasa diferenciación entre los productos disponibles. Esto no solamente influía en una sensación, para el visitante, de una estética profundamente uniforme, sino que además incitaba la formación de un enorme mercado negro en el que se intercambiaban productos occidentales como medias o pantalones vaqueros, en muchas ocasiones adquiridos a los turistas alojados en los hoteles para extranjeros. Esta escasez, en comparación con los estándares de consumo medios en Occidente, era evidente y afectaba a la mayor parte de la ciudadanía de la URSS (Osokina, 2001).

Sin embargo, había otros elementos en la cultura de consumo soviética que causaban mayor perplejidad al visitante, y que tenía que ver con la abundancia y refinamiento de ciertos bienes, particularmente en el terreno de la alimentación. Por ejemplo, el champán se vendía y se bebía en grandes cantidades, y no solamente en los restaurantes más selectos dirigidos a los turistas, sino también las tiendas de alimentación más modestas, donde incluso se vendía por copas desde primera hora de la mañana hasta la noche. Se vendían bocadillos de caviar en prácticamente todas las cafeterías de museos, teatros y salas de conciertos. Y se servían opíparos menús de tres platos y sus correspondientes bebidas incluso en las cantinas más modestas y en pequeños restaurantes locales. En algunas de las tiendas de alimentación más grandes se vendían notables surtidos de decenas de chocolatinas distintas, y diversas tartas y pasteles. Estos ejemplos mostraban una evidencia mayormente desconocida en Occidente, y era que pese a los problemas de desabastecimiento crónicos, con colas kilométricas ante las tiendas y baja calidad de la mayoría de los productos destinados al consumo de la ciudadanía, la cultura de consumo soviética se encontraba también permeada, paradójicamente, de un cierto gusto por el lujo, una suerte de lujo plebeyo o cercano al hombre común, que introducía interrogantes sobre la peculiar conformación de una sociedad de consumo en un lugar donde las modas estimuladas por el mercado estaban, evidentemente, ausentes (Gronow, 2003).

En este artículo, nuestro objetivo va a ser el de tratar de describir el origen y construcción de la cultura del consumo en la Unión Soviética, prestando atención particular a uno de sus ejemplos icónicos, como es la cultura alimentaria. Las políticas culturales eran una parte esencial e importante de la construcción del socialismo en la Unión Soviética. La sociedad socialista, y más aún la próxima sociedad comunista, no solamente exigía la industrialización del país y altos niveles de desarrollo económico y técnico: presumía también la creación de un nuevo tipo de ciudadano, con nuevas y más desarrolladas necesidades y capacidades.

\footnotetext{
2 Jukka Gronow, sociólogo nacido en Finlandia pero con antepasados de origen ruso, relata en el prefacio a su magnífico libro Caviar with champagne (Gronow, 2003) sus visitas a la Unión Soviética, particularmente la primera en el verano de 1968, siendo uno de sus recuerdos más vívidos el de disfrutar, en un café moscovita, de champán soviético y bocadillos de caviar, lujos aristocráticos al alcance de todos los bolsillos en la URSS de Brézhnev (N. del T.).
} 
Esto significó que, en la política soviética, el fomento y cultivo de los gustos de los ciudadanos de la URSS tuviese un papel especial. La cultura alimentaria no fue, evidentemente, una excepción. Nuestro artículo, basado en algunas investigaciones previamente publicadas (ver sobre todo Gronow, 2003; Gronow y Zhuravlev, 2011; Gronow y Zhuravlev, 2015), tiene como base documentación obtenida en diferentes archivos situados en la Federación rusa, que preservan gran cantidad de material de la época soviética. Debido a la naturaleza centralizada de la gestión de la economía en la URSS, se preservaban con celo todos los documentos relacionados con las medidas económicas, desde informes de los comisarios políticos hasta cartas del Ministerio de la Industria Alimentaria a diversas unidades administrativas, tiendas y fábricas locales. Se han consultado archivos ministeriales que, en general, siempre han estado abiertos a los investigadores al contener información técnica y no políticamente sensible. Adicionalmente, se han consultado algunas de las publicaciones periódicas que editaban algunas de las unidades económicas del momento (por ejemplo, Izobilie, traducible como Abundancia, que era la revista del conglomerado de fabricación de bebidas Abrau-Diurso) o las más ideológicas revistas ministeriales, como por ejemplo Organizatsiia i tekhnika sovetskoi torgovli (Organización y técnicas del comercio soviético). Todas estas publicaciones incluían numerosa información en relación a listas de productos y precios, planes y objetivos de producción, campañas políticas y demás. Toda esta información de archivo se ha visto acompañada de fuentes secundarias procedentes de reconocidas investigaciones sociohistóricas y económicas de reputados especialistas en ese período de la Unión Soviética.

Este texto se dividirá en tres secciones. En la primera, hacemos referencia a la peculiar y original construcción de la sociedad de consumo soviética, en pleno estalinismo. En la segunda, nos concentramos en el espacio concreto del consumo alimentario, con referencia en particular a dos cuestiones: el desarrollo de los servicios de restauración y algunos de los bienes asociados a esta nueva cultura de consumo, como el champán; finalmente, la tercera sección se dedica a concluir el texto dando cuenta de algunos de los avatares posteriores de esta peculiar sociedad de consumo.

\section{EL NACIMIENTO DE LA SOCIEDAD DE CONSUMO SOVIÉTICA}

Los historiadores especializados en la Unión Soviética han documentado que, en la década de los treinta del siglo pasado, se produjo una importante transición dentro de la política cultural de la URSS (ver Stites, 1992; Fitzpatrick, 1999; Timasheff, 1946). Tras un período previo en el que se había ensalzado, en la vida pública, una determinada cultura propia del proletariado -caracterizada por un ideal ascético asociado al trabajador que se sacrificaba por la revolución-, a mediados de los años treinta se empezaron a observar cambios notables que tenían, evidentemente, una relación directa con las transformaciones sociales derivadas de la consolidación del estalinismo. De forma progresiva, y tal como ya denunciaran en su momento muchos comunistas, incluido el propio León Trotsky, en el socialismo estalinista estaba emergiendo una nueva clase burocrática, lo que establecía un nuevo orden social jerárquico en el que se pasaban a permitir diferencias culturales y de gustos entre las personas. La consolidación de dicha clase social era el resultado de los programas de industrialización organizados dentro de los planes quinquenales, con los que el Estado soviético debía cumplir la obligación no solamente de alimentar y vestir a la población sino de superar, en términos tecnológicos e industriales, al capitalismo. Nada podía oponerse a este camino, y se utilizó el terror como un recurso disciplinario para alcanzar los objetivos marcados (Hoffman, 1993). Además de una oleada de represión sin precedentes frente a los traidores que saboteaban los objetivos marcados por el Partido y Stalin, se marcaron una serie de líneas específicas destinadas a incrementar la producción industrial. El progreso 
técnico y económico exigía contar con técnicos preparados y con trabajadores dispuestos a alcanzar ciertos objetivos de producción, lo que tuvo como consecuencia el progresivo abandono de los ideales ascéticos e igualitarios que habían dominado hasta entonces el imaginario soviético.

Por otra parte, el extraordinario coste humano del Primer Plan Quinquenal, que había colectivizado la agricultura y puesto el énfasis en una industrialización frenética concentrada en particular sobre la industria pesada, había tenido consecuencias importantes en la forma en que la nueva sociedad socialista empezaba a consolidarse en la URSS. Las diferencias sociales empezaron a aumentar, paradójicamente, dentro de un sistema económico ahora orientado a alcanzar resultados satisfactorios en un contexto de planificación económica total. La industrialización debía, así, completarse con éxito, y ello exigía personal cualificado y motivado. Una generación de técnicos con educación superior procedentes de las clases campesinas y proletarias pasó a desarrollar carreras profesionales en la industria en la década de los treinta, reemplazando a la anterior intelligentsia burguesa (que había sufrido una dura represión a partir del juicio propagandístico de Shajty en 1928) y experimentando, así, una movilidad social ascendente; de este modo, pasan a convertirse, para el régimen, en el nuevo grupo social de referencia frente al proletariado (Fitzpatrick, 1999). Por otra parte, se estimularon comportamientos individualistas en el espacio de la fábrica, con una promoción del productivismo personificada en el modelo estajanovista, que idealizaba el productivismo individualista frente al énfasis anterior en las brigadas de trabajo, de espíritu colectivo. Los objetivos que se perseguían por parte de las autoridades del Partido eran los de estimular la productividad para alcanzar, en distintos sectores productivos, objetivos fantasiosos (Thurston, 1993). Ante los fracasos en cumplir con dichos objetivos, el régimen, que contaba con un poder omnímodo resultado de un totalitarismo asfixiante pero que, al mismo tiempo, se sentía vulnerable ante la opinión de las masas, reaccionó tácticamente. a través de una doble vía: por un lado, culpando a traidores y saboteadores de esa imposibilidad de alcanzar los objetivos (lo que provocó durante toda la década purgas continuas, cuya máxima expresión fueron los Procesos de Moscú); y por otro, persiguiendo, sin pausa, el mostrar a las masas que sus estándares de vida habían efectivamente mejorado. Ante la incapacidad de lograr efectivamente mejorar el nivel de vida del pueblo soviético de un modo general y coherente, al menos se pretendía alcanzar éxitos parciales mediante la provisión de ciertos bienes de consumo específicos, cuyo prestigio simbólico hiciera sentir que la prosperidad, de alguna forma, había llegado finalmente a la Unión Soviética.

De este modo, la necesidad de premiar y castigar dentro del socialismo a aquellos que contribuían o frenaban el desarrollo de la industria soviética fue la base de una serie de medidas políticas y económicas, que contribuirían al aumento de las desigualdades sociales. Ello vino además acompañado por otros cambios significativos, como cambios legislativos que permitían el regreso de los títulos académicos, los rangos en el ejército o las distinciones honorarias (por ejemplo el título de "Héroe de la Unión Soviética") abolidas en la década anterior, o una política de mayor estratificación de los ingresos basada en factores como el lugar de trabajo y el espacio geográfico (permitiendo más ingresos en Moscú y otras ciudades grandes en comparación con lugares más pequeños) (Gronow, 2003). En general, la primera mitad de la década de los treinta significó, fundamentalmente, un abandono de los ideales igualitarios en la URSS, cuya cultura material estaría, a partir de entonces, dominada por una clase media soviética con empleos cualificados y que, de acuerdo con la visión del estalinismo, necesitaría una cultura de consumo acorde a este nuevo escenario, que la incentivase de alguna manera a involucrarse en la construcción del socialismo en un solo país.

Por tanto, y como han señalado algunos autores (Fitzpatrick, 1999; Hessler, 2004), en esa etapa se produce una "transformación de los valores" culturales de gran calado. No solamente está despegando un nuevo modelo económico basado en la industrialización a ultranza, 
sino que, paralelamente, el régimen va a propiciar un cambio de cultura en términos de la relación del ciudadano soviético respecto a la cultura y al consumo. El Partido abandonará la línea de arte proletario para convertir en obligatorio el realismo socialista, favoreciendo por un lado un neoclasicismo inspirado en la alta cultura clásica (promocionando el ballet, la pintura didáctica o la música clásica, y rechazando los estilos vanguardistas) y, a la vez, una cultura de masas que incluía novelas de entretenimiento, películas, o música de bandas militares accesibles para todos (Stites, 1992). Paralelamente se persigue la construcción de una sociedad de consumo que sirva para colmar las aspiraciones de esa nueva clase media compuesta por burócratas y profesionales. Estas personas viven bien y están ganando dinero, pero no hay nada en qué gastar dicho dinero. El desarrollo de nuevos productos con mayor calidad era, de acuerdo a algunos autores (Fitzpatrick, 1999; Dunham, 1976), una aspiración de esas nuevas élites soviéticas, que quieren visibilizar de algún modo su nuevo estatus social. Sin embargo, este análisis es, a nuestro juicio, incompleto. Como ya se ha argumentado en otro trabajo (Gronow, 2003), la realidad es que, pese a que los principales consumidores de estos nuevos productos de calidad fuesen, a la larga, los miembros de esa nueva intelligentsia, detrás del impulso a esas nuevas industrias latía la gran promesa de la abundancia socialista, y en la mente de los creadores y planificadores de los productos de consumo, no aparecían como destinatarios los miembros más afortunados de la sociedad sino todos los ciudadanos soviéticos leales, decentes e industriosos.

Un objetivo fundamental en la creación de dicha sociedad de consumo era la de proyectar una imagen diferente de la sociedad soviética, que hasta entonces había sufrido, en particular tras el Primer Plan Quinquenal y la colectivización de la tierra, situaciones de total desabastecimiento en los comercios. La producción, orientada hacia el desarrollo de la industria pesada, marginaba la fabricación de bienes de consumo, los cuales eran de muy mala calidad y se limitaban a cubrir las necesidades básicas sin preocupación alguna por la estética. Las crónicas sobre la uniformidad de los vestidos soviéticos, su baja calidad, y su grisura y tristeza, eran frecuentes tanto por parte de observadores extranjeros (ver Gidé, 1937) como de los propios dirigentes del régimen (Mikoyán, 1971), y esta opinión se extendía a prácticamente todos los (pocos) objetos de consumo disponibles. Sin embargo, desde mediados de la década de los treinta, hay un intento por revolucionar los estándares de calidad de los productos, de forma que pudieran rivalizar con los occidentales y así mostrar al mundo que el trabajador soviético podía también aspirar a una vida mejor y más cómoda.

Un momento decisivo aquí va a ser el año 1936, cuando Stalin acuñó un famoso eslogan: "La vida se ha vuelto mejor, la vida se ha vuelto más feliz, camaradas" (Petrone, 2000; Gronow, 2003). Como se anunciaba en ese momento, la Unión Soviética abandonaba oficialmente el racionamiento, lo que parecía implicar que había alcanzado, por fin, una etapa de abundancia generalizada, y sus ciudadanos, tras una larga espera, podían finalmente disfrutar del bienestar material tras años de sacrificio. Coincidiendo de forma irónica y trágica con una ola de represión política extraordinaria, las autoridades soviéticas propagaron entre la población, durante la segunda mitad de la década de los treinta, la idea que la felicidad debía sustituir al sacrificio y al ascetismo, organizando así numerosos desfiles y celebraciones populares en los que se consumían bebidas y alimentos y se disfrutaba del baile, en una atmósfera relajada (Petrone, 2000). La organización de buena parte de estas nuevas políticas destinadas a la felicidad de los habitantes del pueblo recaerían en varios dirigentes, en particular Anastás Mikoyán, que desde el Comisariado de la Industria Alimentaria desarrollaron un proyecto histórico, que es realmente un caso único: la construcción de una cultura material desde la nada, en la que absolutamente todas las decisiones relacionadas con la producción de cualquier objeto pasaban a tomarse desde las altas esferas del gobierno y la administración (Gronow, 2003).

Este anuncio de Stalin llegaba tras un período bastante duro para los habitantes del enorme país socialista, que habían pasado por varias etapas en lo que a patrones de consumo 
se refiere. Así, durante la Guerra Civil después de la Revolución, el bando bolchevique introdujo el llamado "Comunismo de guerra", que implicó la confiscación de alimentos y que, combinado con las inclemencias del conflicto, provocaron situaciones de hambre a gran escala. La NEP (Nueva Política Económica) de mediados de la década de 1920 permitió una cierta mejora en las condiciones de vida al permitir mercados limitados de alimentos, pequeñas explotaciones agrícolas, tiendas privadas y restaurantes a pequeña escala; no obstante, en la transición a la década siguiente y coincidiendo con la puesta en marcha del Primer Plan Quinquenal, se liquidaron todas las "tiendas comerciales" y cooperativas, imponiendo un estricto racionamiento centralizado de alimentos. En este nuevo sistema, todos los alimentos se distribuyeron de acuerdo con un sistema de nomenklatura, que clasificaba a toda la fuerza laboral de acuerdo con su importancia económica, geográfica y política. Los efectos se hicieron notar de forma dramática. Tras las incautaciones de alimentos por parte del Estado y la colectivización forzosa del campo, la población campesina y rural pasó a vivir casi en un nivel de pura subsistencia que, a principios de la década de los treinta, culminó de forma catastrófica con una gran hambruna que provocó millones de muertes (golpeando de forma particularmente intensa a los campos y aldeas de Ucrania), y generando incluso problemas de abastecimiento en las grandes ciudades. Salir del círculo vicioso del racionamiento se convirtió en una prioridad para las autoridades soviéticas, y Stalin percibió que la puesta en marcha de una sociedad de consumo incipiente podría ser un buen "acuerdo" entre el poder y la nueva generación de técnicos (educados y urbanitas) y trabajadores cualificados.

Como las autoridades e ideólogos soviéticos insistieron en repetir en esos momentos, la Unión Soviética había dejado de ser por fin una "sociedad de cuartel", donde todas las personas vivían de la misma forma y compartían los escasos recursos destinados al consumo, y pasar a una nueva fase en la construcción del socialismo caracterizada por una mayor abundancia. Con un mayor bienestar material, las necesidades de las personas también aumentaron, y se desarrollaron de forma consecuente. Surgieron de hecho nuevas necesidades, más elevadas y más cultivadas, para sustituir a los antiguos y muy austeros estándares. Sin embargo, en la propaganda soviética solo se justificaban aquellas necesidades que eran racionales y que contribuían al desarrollo de una personalidad armoniosa y equilibrada, una que podría hacer una valiosa contribución a la construcción del socialismo. De este modo, todos los deseos que fuesen "excesivos" pasaban a ser vistos como perjudiciales: uno tenía que aprender a ser moderado, y evitar los extremos. En última instancia, el Partido Comunista o sus representantes, los funcionarios superiores del Partido (a través de las instituciones estatales de planificación), pasaron a decidir qué necesidades y bienes eran los apropiados y cuáles serían los excesivos e indeseables (Gronow, 2003).

Este interés por desarrollar nuevos productos de consumo implicó importantes cambios, desde la proliferación de electrodomésticos y equipos de audio, a nuevas prendas de vestir, perfumes ("el regalo de Stalin a las damas"), bicicletas o, de forma particular, una cultura culinaria que apostaba por incrementar la variedad de ciertos productos que podrían ser considerados como placeres. Este era el caso de los chocolates, el caviar, ciertas bebidas alcohólicas como el coñac, el vino y, particularmente el champán. Todos estos productos iban dirigidos a unas nuevas clases medias, que tenían ciertas aspiraciones materiales pero que a la vez compartían, como ciudadanos leales al Partido, una suerte de conciencia cultural (kulturnost) (Attwood, 1999; Kelly y Volkov, 1998) que tenía dos características principales. Por un lado, tenían que mostrar un deseo de realización personal, apareciendo ante los demás como individuos cultivados y capaces de disfrutar de las bellas artes, la música clásica y la literatura y mostrando, además, "buen gusto" en la selección de sus ropas y por supuesto buena conducta. Y por otro lado, se hacía a la vez una llamada a mantener una cierta "modestia", un ascetismo casi puritano (Vainshtein, 1996; Gronow y Zhuravlev, 2015) identificado con la cultura socialista. Esta tensión existente entre una tendencia a 
la estandarización y lo modesto frente a ocasionales resistencias, expresadas en estilos más llamativos mantenía, de forma peculiar, una relación homológica con el campo más amplio de la cultura del consumo soviético, donde en general coexistía la mala calidad, fealdad, pobreza y grisura de casi todos los productos disponibles con ocasionales destellos de belleza y lujo. El ejemplo más clásico es el de la cultura culinaria soviética, donde una extraordinaria estandarización va a acompañarse de la producción de consumos asociados al gusto aristocrático, como el caviar y el champán.

\section{LA CULTURA CULINARIA SOVIÉTICA}

La consolidación del estalinismo tuvo lugar a finales de la década de los veinte, cuando la vieja guardia bolchevique que había acompañado a Lenin fue marginada y derrotada dentro del PCUS por las maniobras de Stalin, que terminó convirtiéndose en el líder absoluto del país. La política alimentaria, así como la política económica en general, experimentaron en ese momento muchos cambios drásticos, que tuvieron su efecto en la provisión y distribución de alimentación. Se optó por una planificación central, de forma que desde mediados de la década de 1930, todos los restaurantes de la URSS pasaron a trabajar bajo la estrecha orientación de la Administración Central de Restaurantes y Cafeterías, dependiente del Comisariado de Comercio. Los menús de los restaurantes, así como todos los platos y bebidas vendidos, pasaron a partir de entonces a ser aprobados oficialmente por el Estado, incluyendo además las cantidades exactas de carne, patatas, repollo, etc. que debía contener cada plato. Así, los órganos estatales controlaban, literalmente, todos los ingredientes, e incluso la cantidad de platos de carne en los menús diarios de los restaurantes y cantinas (Gronow y Zhuravlev, 2011).

Esto no significaba que no pudiera haber una cierta variación y renovación de sus contenidos, debido a la iniciativa de individuos y grupos profesionales activos en el campo de la gastronomía; después de todo, era obvio que en las doctrinas básicas del marxismo-leninismo no se ofrecían muchos consejos concretos o detallados en cuestiones culinarias u otros debates relacionados con el gusto. Una de las peculiaridades de la política alimentaria soviética era la estricta regulación, mediante normas estatales, de las recetas utilizadas en la restauración pública: regulación ésta hasta el último detalle, que especificaba hasta las cantidades de ingredientes que se necesitaban para preparar un plato o producto. Los mismos estándares eran válidos en toda la Unión Soviética, desde Asia Central hasta las Repúblicas Bálticas, y cubrían absolutamente todo, desde vinos y chocolates hasta el repollo o las salchichas. Estas normas estatales se renovaban periódicamente, y las normas para las recetas utilizadas en restaurantes y cantinas se ajustaban en consecuencia. En la década de 1930, la proporción de personas que, en la URSS, disfrutaban de sus comidas principales en el lugar de trabajo y en las cantinas escolares era mayor que en casi cualquier otro lugar del mundo.

Sin embargo, la centralización había venido acompañada de una baja diversidad gastronómica. Como se afirmaba en un estudio, un ejemplo típico era el de la cantina de la planta química en Nizhny Novgorod en 1932: durante los veinticinco días hábiles de febrero, los trabajadores recibieron sopa de col (shchi) bajo diferentes nombres en cinco ocasiones; también hubo borsch, una sopa hecha de col y remolacha, ofrecido otras cuatro veces durante el mes. Otras sopas estaban hechas de guisantes, patatas y pasta. Mientras tanto, el plato principal solo contenía carne y pescado unas seis veces en todo ese mes. Durante el resto del mes, la mitad de los días, para ser precisos, los trabajadores comían simplemente pasta y cereales: alforfón (cuatro veces) o papilla de mijo (cinco veces). Estos menús y la cantina, sin embargo, fueron nominados para el concurso de la mejor cantina de la región. 
La escasez era, así, generalizada, y las materias primas, incluso para algunos de los alimentos más modestos, simplemente no se podían encontrar o, si estaban disponibles, solo era de manera irregular. La comida en un restaurante de primera clase "real" era, en general, algo mejor y más sabrosa que en las cantinas. Estaba compuesta de ingredientes de mejor calidad, muchas de los cuales nunca estaban disponibles en las tiendas de alimentos, sino que solo se distribuían a los restaurantes de primera clase y a unos cuantos comedores pertenecientes a espacios de trabajo altamente privilegiados.

Sin embargo, a pesar del hecho de que los precios eran mucho más altos y, por lo tanto, más caros para los trabajadores soviéticos comunes, la calidad de la comida y el servicio en muchos de los restaurantes más refinados no mejoraba tanto respecto a las cantinas como se podría suponer. Una gran proporción de la población, además, no tenía otra alternativa más que acudir a los servicios de restauración pública durante un período sustancial de sus vidas. Este fue el caso de millones de niños y adolescentes en las denominadas “instituciones estatales cerradas" (orfanatos, internados), así como en los jardines de infancia y escuelas ordinarias: todos ellos recibieron al menos sus almuerzos y refrigerios en las cantinas de sus comedores educativos. Otros dos grandes grupos de población, los que realizaban el servicio militar y los presos en diversas instituciones penitenciarias, desde las cárceles hasta los campos de trabajo, se vieron obligados a confiar más o menos totalmente en los servicios de restauración pública para satisfacer sus necesidades nutricionales básicas. La comida servida y los estándares de servicio en todas estas instituciones "cerradas" fueron, por regla general, de muy mala calidad. En el mejor de los casos, podría decirse que garantizaba el mínimo de necesidades nutricionales. Existían además otras carencias. Las cantinas, en su mayoría, no disponían de cuchillos y tenedores, proporcionando únicamente cucharas de aluminio. Presentaban así un marcado contraste con los ideales y modelos representados por los mejores restaurantes soviéticos y con las guías culinarias oficiales de la época.

Y es que, frente a esta estandarización masiva y de baja calidad, tan representativa del consumo soviético, la cúpula del partido con Stalin a la cabeza quería promover el desarrollo de un consumo más atractivo, y por supuesto el campo alimentario no fue una excepción. El dirigente del PCUS Anastás Mikoyán fue particularmente activo en el desarrollo de esta planificación de las necesidades (si bien el propio Stalin se implicó personalmente en algunas ideas y sugerencias). Mikoyán fue decisivo particularmente en lo que fue el diseño de los modelos e ideales del consumo de alimentos en la URSS, así como del consumo en general, gracias a sus viajes a diversos países (Francia, Estados Unidos) de donde obtuvo inspiración para el diseño y desarrollo de productos específicos. Un caso muy llamativo es, sin duda, el relacionado con los signos tradicionales de lujo: el champán, los chocolates y el caviar, que se convirtieron, a fines de la década de 1930, en una parte esencial de la cultura soviética. El objetivo era que algunos elementos de lo que había sido la representación simbólica del consumo ostentoso de la vieja aristocracia rusa se convirtieran en un producto masivo, accesible para el proletariado (Gronow, 2003). Esto supuso que se hiciesen esfuerzos enormes por parte del Comisariado dirigido por Mikoyán para desarrollar nuevas industrias alimentarias, priorizándose por parte de las autoridades la inversión en productos como los chocolates, el caviar, los vinos de calidad, helados, coñac, champán, etc. Estos productos, que estarian disponibles en tiendas especializadas como las nuevas Gastronom y Bakaleia, ideadas por Mikoyán, también se servirían en restaurantes, los cuales empezaron a servir menús con tres platos, o en cafeterías de inspiración vienesa donde se pudiese disfrutar de una taza de café y un trozo de pastel. Estos nuevos hábitos se convirtieron en modelos, para las clases urbanas, en términos culinarios (Rothstein y Rothstein, 1991). El régimen pasó a anunciar, regularmente, que una nueva época había llegado, y en el Segundo Plan Quinquenal se planificaron grandes cifras de producción que, a la larga, se demostraron poco realistas. 
Sin embargo, hubo algunas tensiones interesantes, integradas en estos ideales ahora ansiosamente propagados de la buena vida. En primer lugar, estos productos en teoría deberían estar disponibles para todos al mismo tiempo, o al menos para todos los ciudadanos decentes y trabajadores de la Unión Soviética y no, como en el caso del capitalismo, solo para los pocos miembros de la clase dominante. En la práctica, sin embargo, estos consumos de mayor calidad solamente estaban, en muchos casos, disponibles para la vanguardia urbana e industrial de la clase obrera soviética, así como para sus autonombrados representantes, que era por supuesto la élite gobernante del Partido Comunista, la nomenklatura, y otros altos funcionarios públicos, casi todos ellos residentes en Moscú y otras grandes ciudades. Y en segundo lugar, estos lujos deberían, idealmente, ser producidos en masa, esto es, a nivel industrial. Por supuesto, hubo problemas en lo relativo al servicio a las masas trabajadoras en los mejores restaurantes o cafés, pero en lo que respecta a los productos alimenticios, al menos ya no existía ninguna contradicción entre el aura de rareza o exclusividad, generalmente característica del lujo "burgués", y las versiones masivas y baratas de tales artículos. En el socialismo, estos lujos estarían disponibles para todos, a un precio eso sí barato. En la nueva "estética de los productos básicos" soviéticos oficiales, el hecho de que los bienes se produjeran industrialmente según las últimas técnicas se convirtió, de hecho, en un valor añadido adicional y un signo de finura socialista, en lugar de un marcador de inferioridad cultural y social como sucedía en los regímenes de consumo del capitalismo (Gronow, 2003).

Además, ya desde finales de la década de 1920, nuevas ideas progresistas y científicas de la nutrición habían empezado a dominar el discurso soviético sobre una alimentación adecuada. En 1929 se abrió en Moscú un Instituto Científico de Nutrición. Los expertos nutricionistas soviéticos expresaron gran confianza en el desarrollo de nuevas soluciones científico-técnicas para los problemas de nutrición. Promovieron enormes cocinas y comedores de fábrica estandarizados como soluciones ideales para atender efectivamente las necesidades nutricionales básicas de la población trabajadora. Del mismo modo, pusieron grandes esperanzas en el desarrollo de nuevos alimentos sintéticos producidos industrialmente, como los sustitutos de proteínas derivados de las habas de soja. Los representantes radicales de la ciencia natural moderna desafiaron los cánones tradicionales de nutrición, y pidieron una nueva dieta racional contrastada científicamente, basadas en el nuevo conocimiento de las cantidades adecuadas de calorías y vitaminas necesarias para mantener el cuerpo humano saludable y poder realizar en condiciones el trabajo. Para ellos, el arte culinario y los placeres sensuales asociados al acto de comer eran un "anacronismo del pasado burgués". Por ejemplo, M. Dubyanskaya afirmó en su libro Zdorovaya pishcha i kak yeyo gotovit (Alimentos saludables y cómo cocinarlos) que el uso de especias y vinagre eran hábitos poco saludables y restos del pasado que solo conducían a comer en exceso. En las instrucciones culinarias en la prensa popular, la nueva ciencia de la nutrición, sin embargo, en su mayoría unió fuerzas con la antigua gastronomía. A partir de 1923, la revista mensual de mujeres trabajadoras soviéticas Rabotnitsa comenzó a publicar recetas de alimentos regulares bajo el título general domovodstvo (limpieza). A pesar de la popularidad de la nueva doctrina de la nutrición racional, la revista también publicó recetas bastante elaboradas de la cocina aristocrática "pasada de moda". Es bastante notable que el nuevo énfasis puesto en el buen gusto de los alimentos coincidiera con la colectivización de la agricultura y el establecimiento de comedores colectivos en los koljoses, a finales de los años veinte y principios de los treinta. Por ejemplo, en su libro de cocina dirigido a cocineros profesionales en los comedores del koljós, la Sra. Zarina enfatizó la necesidad de prestar especial atención al sabor de los alimentos, ya que el buen sabor fomentaba el buen apetito y la mejor absorción de nutrientes en el cuerpo humano. Prestar atención al sabor de la comida y al placer culinario no se consideraba, por lo tanto, necesariamente como un signo 
de una actitud burguesa reaccionaria. La nueva ciencia de la nutrición desempeñó un papel importante, pero limitado, en la ideología y la política de los alimentos a lo largo de la era soviética (Gronow y Zhuravlev, 2011).

Lo cierto en todo caso es que Mikoyán y otros líderes del estalinismo fueron fundamentales en la construcción de la cultura alimentaria soviética, siendo el gusto de dichos líderes una variable muy concreta que acababa afectando a la vida cotidiana de sus ciudadanos. El propio Mikoyán, Comisario del Pueblo para la Industria Alimentaria, señalaba que no se permitía la producción de ciertos alimentos en ninguna empresa antes de que dicho producto se fabricase a su gusto. Afirmaba con rotundidad: "iLos probé todos!". En este sentido, el champán soviético fue probablemente el mejor ejemplo de este "cambio de valores". La producción en masa de champán también comenzó a mediados de la década de 1930, y sus promotores estaban totalmente convencidos de que, debido a la moderna tecnología utilizada en su producción, no solo tenía la misma calidad que su modelo francés, sino que a menudo incluso lo superaba gracias a su excelente sabor y otras cualidades organolépticas. Resulta enormemente interesante observar que, tras unos años en los que la desnutrición y las hambrunas habían sido la tónica constante, las autoridades soviéticas, con el liderazgo de Stalin, hubiesen dedicado su esfuerzo a la producción de vino espumoso. Las autoridades soñaban con mostrar destellos de ese paraíso futuro que estaba por venir en un contexto marcado por la escasez, y para ello asumieron medidas de calado extraordinario y muy caras económicamente para conseguir alcanzar dicho objetivo. Stalin dio su bendición y Mikoyán se implicó personalmente en el desarrollo de la industria del champán, visitando Francia como inspiración y reorganizando las fábricas bajo una dirección centralizada. Nada ni nadie se interpondría en ese camino, lo que costó purgas y detenciones en la empresa pública vinícola: este esfuerzo ni siquiera se detendría durante la II Guerra Mundial. El resultado fue que, a finales de los años treinta, el champán soviético se había convertido en un objeto de consumo muy popular (aunque no se alcanzaran ni de lejos los objetivos de producción), estando disponible, al menos en las grandes ciudades, antes de las grandes celebraciones. Representaba, quizá como ningún otro bien, la "buena vida" aunque pronto se hicieron esfuerzos similares con otros productos de lujo (Gronow, 2003).

En el sector de la restauración, mientras tanto, en el que la racionalización técnica no se podía conseguir tan fácilmente al no poder aplicarse los principios de mecanización y estandarización que operaban en la industria alimentaria, la tendencia general era otra: construir grandes espacios, grandes comedores en los lugares de trabajo, grandes salas de restaurantes con cientos de mesas, o grandes cafés. Sin embargo, lo que había cambiado, en comparación con la década de 1920, era que los comedores de gran tamaño y de trabajo pasaron a ser el nivel más bajo de la restauración soviética. Desde mediados de la década de 1930 estuvieron acompañados por restaurantes y cafés más caros, que contaban con productos y servicios de mayor nivel. En algunos de estos espacios, hubo una oferta regular de algunos de los productos antes citados como el champán, el caviar y otros productos, además de generosos menús de tres platos. Algunos bares de autoservicio y cervecerías, donde los clientes consumían alimentos y bebidas de pie, se sumaron a la lista de las instalaciones soviéticas de restauración, siendo su menor tamaño compensado por una rotación de clientes más rápida.

Después de la Segunda Guerra Mundial, se hizo además un esfuerzo por ampliar la paleta de gustos con la apertura a otras gastronomías. En la cocina soviético-rusa, la cocina caucásica, y en particular la cocina georgiana desempeñó un papel muy similar al de la cocina mediterránea en Europa occidental, presentando una gastronomía étnica sabrosa, picante y algo exótica como complemento de la cocina ruso-europea. Estos platos se servían también en los mejores restaurantes soviéticos. Las ciudades soviéticas más grandes tenían, 
a menudo, uno o dos restaurantes caucásicos especiales que contaban con un clásico menú caucásico, así como vinos georgianos y aguardientes armenios en su lista, aunque esto no significaba que siempre estuvieran disponibles para la venta. Moscú tenía toda una cadena de restaurantes étnicos; de hecho una para casi todas las repúblicas soviéticas inauguradas en las décadas de la posguerra, además de los restaurantes georgianos, armenios, azeries, uzbekos, ucranianos y bielorrusos. También tenía una cadena de restaurantes dedicados a las cocinas de los países socialistas hermanos, como los restaurantes Praga, Budapest, Habana o Pekín. Este último se convirtió en un espacio problemático después de que el cisma entre la Unión Soviética y China se profundizara en los años cincuenta: no se cerró, pero pasó a servir comida del Lejano Oriente ruso. Otras ciudades soviéticas, incluidos centros tan importantes como Leningrado o Kiev, tenían que contentarse con un restaurante genérico europeo, aunque Kiev pudo presumir de un restaurante alemán, llamado Leipzig, que en los años sesenta y setenta sirvió, entre otros platos, los famosos filetes empanados Schnitzels, acompañados por cerveza checa de la marca President (Gronow y Zhuravlev, 2011).

Asimismo, la publicación intensiva de libros de cocina, tanto generales como enfocados en especialidades nacionales en los decenios de 1950 y 1960, tanto en ruso como en otros idiomas que se hablaban en la Unión Soviética, sin duda contribuyó enormemente a la unificación de los hábitos alimenticios soviéticos al ofrecer un repertorio estándar, pero también amplio, de cocina y hábitos culinarios a todos los ciudadanos soviéticos interesados a lo largo y ancho del vasto país, independientemente de sus orígenes étnicos o nacionales (Piretto, 2009). También contribuyó a la diversificación gradual de la cocina soviética, ofreciendo nuevas recetas étnicas e internacionales más exóticas. La naturaleza centralizada del sistema soviético de distribución de alimentos y servicio de comidas, con su limitada cantidad de alternativas y su preferencia por los platos producidos industrialmente, ciertamente supuso una barrera al espíritu innovador de muchos cocineros aficionados, y llevó a una cierta uniformidad forzosa en los hábitos alimenticios de la URSS. Las alternativas a la comida soviético-rusa estándar se promocionaron del mismo modo, de forma que la imagen común de otras cocinas étnicas y regionales entre los consumidores soviéticos ordinarios fue probablemente, al menos, tan estereotipada como en los mercados de alimentos más comerciales del occidente capitalista.

En todo caso, fue obvio que el mercado y la cultura alimentarios soviéticos se fueron haciendo cada vez más variados, y que tanto la disponibilidad como la calidad de los alimentos mejoraron. Los ciudadanos soviéticos también tuvieron la oportunidad de degustar algunos alimentos y bebidas extranjeros después de la Guerra, desde el queso finlandés hasta la cerveza checa e incluso la Pepsi-Cola norteamericana. En todo caso, la proporción de las importaciones de alimentos, excepto materias primas, fue bastante modesta a lo largo de toda la era soviética (Gronow y Zhuravlev, 2011). En general, la población de la URSS se enfrentó, de forma cotidiana, a una escasez atávica de bienes de consumo y a una estandarización de ciertos bienes en un formato muy poco atractivo, que ocasionalmente se compensaba con la puesta a la venta a precios asequibles de ciertos productos de mayor calidad que, en todo caso, rara vez estaban disponibles fuera de las grandes ciudades.

\section{CONCLUSIÓN}

Como hemos señalado anteriormente, la cultura del consumo soviética no ha sido siempre tan gris y aburrida como generalmente se ha descrito, al menos en Moscú y otras grandes ciudades. La creación de una economía de bienes de lujo es un ejemplo de que existían importantes matices y complejidades en la sociedad de consumo que estaba siendo 
promovida por el estalinismo. Muchos productos relativamente atractivos y sin embargo asequibles se comercializaron y estuvieron disponibles para el consumidor ordinario. Es importante señalar además el elemento kitsch presente en esta peculiar situación: los trabajadores soviéticos, que viven en la pobreza y sufren unos déficits permanentes en prácticamente todo lo relacionado con los estándares de consumo ordinario, tienen a la vez a su alcance productos como el champán o el caviar que, en Occidente, se encuentran fuera del alcance del obrero capitalista de la época (Gronow, 2003). El hecho de que la economía fuese planificada y absolutamente todo lo susceptible de ser producido se decidiese en las esferas de la dirigencia implicaba que no existiese una economía que conectase como en el mercado necesidades y producción, lo que conllevó, en muchos casos, decisiones como la fabricación de champán, que implicaban movilizaciones masivas de recursos de otras áreas, generando importantes problemas de asignación de recursos y, habitualmente, una enorme insatisfacción entre la población. Esta culpaba, con enorme resignación, de estos problemas al Partido / Estado que, a su vez, buscaba chivos expiatorios en diversos traidores y saboteadores, sobre todo durante el período estalinista (Thurston, 1993).

Las reglas de esta economía se mantuvieron completamente ajenas a los ritmos y tendencias existentes en el capitalismo, donde las presiones de la moda y la necesidad de obsolescencia eran cruciales para el funcionamiento del sistema de mercado. En el espacio socialista no existían modas en el sentido estricto del término, sino que eran las autoridades las que interpretaban qué debía fabricarse para satisfacer las necesidades de la población. Simmel (1991) argumentaba que la moda era un síntoma de la modernidad por excelencia, donde los procesos de imitación social y diferenciación individual se reforzaban mutuamente, lo que permitía una mediación entre lo social y lo individual; en las sociedades socialistas, esta tensión se organizaba, sin embargo, de forma muy diferente. De ahí el impulso desde las autoridades a la creación de una esfera de cultura material que representaría una nueva forma de vida socialista, aunque esta concesión al individualismo siempre se encontró con importantes frenos.

Aunque Zygmunt Bauman (1990/1991) ha argumentado que el desarrollo de necesidades y demandas por parte de los consumidores fue un elemento decisivo en la disolución del socialismo, este no fue exactamente el caso de la URSS de Stalin, porque el proceso de individualización se encontraba constreñido por otras normas sociales. Por ejemplo, la kulturnost o decencia implicaba evitar ciertas desviaciones respecto a una norma general de "buenas maneras"; la lógica de los favores internos, casi de clan, en la distribución de lo poco disponible también generaba adhesiones y cierres grupales; $y$, finalmente, prácticamente todo lo ofertado respondía a lo que Stalin y sus lugartenientes definían como "lo que debería ser el gusto de la gente corriente", lo que generaba un enorme control sobre las posibles diferencias que el sistema permitía desplegar, reduciendo las expresiones individuales a un límite. Al final, la conformidad fue un elemento clave en la sociedad de consumo soviética, aunque incluso esto debe ser matizado. La ciudadanía soviética, en su mayoría, no eran creyentes en el comunismo ni cínicos que habían perdido la fe en todas las promesas de las autoridades. Los que tuvieron la oportunidad trataron de disfrutar de los lujos que se les ofrecían a la vez que soportaban la escasez en otros aspectos de la vida, con la idea de la esperanza en un futuro mejor o, simplemente, ante la imposibilidad de poder hacer otra cosa (Gronow, 2003).

Lo cierto es que el estalinismo fue capaz de impulsar una sociedad de consumo peculiar, diferenciada desde luego de la existente en el mundo capitalista, y por supuesto plagada de contradicciones. Tras el fin del racionamiento y los enormes sacrificios asociados a la reestructuración de la economía soviética, las autoridades declararon que habían llegado tiempos mejores, y pusieron todo su esfuerzo en prometer un mejor nivel de vida. Esto exigió 
grandes proyectos, propaganda y anuncios para crear nuevos productos que literalmente no existían en aquel momento, para satisfacer a un mercado enorme de 150 millones de personas. Esto evidentemente, era casi imposible de cumplir, pero sirvió para que al menos una serie de productos peculiares y lujosos pasaran a fabricarse y se convirtieran en asequibles para la población. Y al mismo tiempo, generó una serie de tensiones importantes dentro del socialismo, ante el riesgo inherente de una restauración del modelo mercantil. Y es que al final, la satisfacción de los deseos de los consumidores implicaba riesgos importantes para el sistema, que amenazaban los objetivos básicos del socialismo soviético tal y como se había constituido: control del Estado de los medios de producción, igualdad ente los ciudadanos y seguridad militar dentro del país. Sin duda, un campesino o un comerciante implicado en esa mejora de los productos de consumo podía llevar, en su interior, el alma de un corrupto capitalista; además, si se permitía una mayor capacidad de elección, el Estado sería incapaz de satisfacer las necesidades diferenciadas de sus habitantes. Estas contradicciones entre mantener un control centralizado de la producción de recursos y permitir las diferencias en el consumo marcaron la sociedad de consumo soviética, perfilando de forma evidente los límites de un modelo que, finalmente, rara vez pudo cumplir unas promesas habitualmente poco realistas.

\section{REFERENCIAS BIBLIOGRÁFICAS}

Attwood, L. (1999). Creating the New Soviet Woman: Women's Magazines as Engineers of Female Identity, 1922-53. Basingstoke: Macmillan. https://doi.org/10.1057/9780333981825

Bauman, Z. (1990/1991). Communism: A Post Mortem. Praxis International, 10(3/4): 185-192. https://doi.org/10.1177/0725513609353713

Dunham, V. (1976). In Stalin's Time. Cambridge: Cambridge University Press.

Fitzpatrick, S (1999). Everyday Stalinism. Ordinary Life in Extraordinary Times. Soviet Russia in the 1930s. Oxford: Oxford University Press.

Gide, A. (1937). Back from the USSR. Londres: Martin Secher and Warburg.

Gronow, J. (2003). Caviar with Champagne. Common Luxury and the Ideals of the Good Life in Stalin's Russia. Oxford: Berg. https://doi.org/10.5040/9781350050747

Gronow, J. y Zhuravlev, S. (2011). The Book of Tasty and Healthy Food: The Establishment of Soviet Haute Cuisine. En J. Strong, (ed.) Educating Tastes. Food, Drink, and Connoisseur Culture (pp. 24-57). Lincoln: University of Nebraska Press.

https://doi.org/10.2307/j.ctt1df4fx7.5

Gronow, J. y Zhuravlev, S. (2015). Fashion meets Socialism. Fashion Industry in the Soviet Union after the Second World War. Helsinki: SKS. https://doi.org/10.21435/sfh.20

Hessler, J. (2004). A Social History of Soviet Trade. Trade Policy, Retail Practices and Consumption, 1917-1953. Princeton: Princeton University Press. https://doi. org/10.1515/9781400843565

Hoffman, D. (1993). The Great Terror on the Local Level: Purges in Moscow Factories, 19361938. En J. A. Getty, R. T. Manning (Eds.), Stalinist Terror. New Perspectives (pp. 163-167). Cambridge: Cambridge University Press. https://doi.org/10.1017/CB09780511626012.008

Kelly, C. y Volkov, V. (1998). Directed Desires: Kul'turnost' and Consumption. En C. Kelly, D. Shepherd (Eds.), Constructing Russian Culture in the Age of Revolution: 1881-1940 (pp. 291-313). Oxford: Oxford University Press. 
Mikoyán, A. (1971). “Dva mesiatsa v SShA” SShA. Ekonomika, politika, ideologiia, 10.

Osokina, E. (2001). Our Daily Bread: Socialist Distribution and the Art of Survival in Stalin's Russis, 1927-41. Armonk, NY: Sharpe.

Petrone, K. (2000). Life Has Become More Joyous, Comrades: Celebrations in the Time of Stalin. Bloomington: Indiana University Press.

Piretto, G. P. (2009). Tasty and Healthy: Soviet Happiness in one Book. En M. Balina, E. Dobrenko (eds.), Petrified Utopia. Happiness Soviet Style (pp. 79-96). Londres: Anthem Press. https://doi.org/10.7135/UP09781843318170.006

Rothstein, H. y Rothstein, R. A. (1991). The Beginnings of Soviet Culinary Arts. En M. Giants, J. Toomre (eds.), Food in Russian History and Culture (pp. 162-176). Bloomington: Indiana University Press.

Simmel, G. (1991). Fashion. En G. B. Sproles (ed.), Perspectives on Fashion (pp. 130-155). Minneapolis: Burgess Publishing.

Stites, R. (1992). Russian Popular Culture. Entertainment and Society since 1900. Cambridge: Cambridge University Press.

Thurston, R. (1993). “The Stakhanovite Movement: The Background to the Great Terror in the Factories, 1935-1938". En J. A. Getty, R. T. Manning (Eds.), Stalinist Terror. New Perspectives (pp. 142-160). Cambridge: Cambridge University Press. https://doi.org/10.1017/CBO9780511626012.007

Timasheff, N. S. (1946). The Great Retreat: The Growth and Decline of Communism in Russia. Nueva York: E. P. Dutton and Company.

\section{NOTA BIOGRÁFICA}

Jukka Gronow es Emeritus Professor en el departamento de investigación social de la Universidad de Helsinki. Especialista en sociología del consumo, entre sus obras más destacadas se incluyen Caviar with champagne (Berg Publishers, 2003), Fashion meets socialism (SKS, 2015, en co-autoría con Sergey Zhuravlev) y Deciphering Markets and Money: A Sociological Analysis of Economic Institutions (HUP, 2020)

Sergey Zhuravlev es Professor of History en el Instituto de Historia Rusa de la Academia Rusa de Ciencias, Moscú. Es especialista en historia rusa de los siglos XX y XXI. Entre sus trabajos destaca Fashion meets socialism (SKS, 2015, en co-autoría con Jukka Gronow). 